\title{
Peroxisomal heat generation and possible relationship with peroxisomal disorders
}

\author{
Anna Petroni \\ Dept. of Pharmacological and Biomolecular Sciences, University of Milan, Milan, Italy
}

\section{Email address:}

anna.petroni@unimi.it (A. Petroni)

\section{To cite this article:}

Anna Petroni. Peroxisomal Heat Generation and Possible Relationship With Peroxisomal Disorders. Advances in Biochemistry. Vol. 1, No. 1, 2013, pp. 5-6. doi: 10.11648/j.ab.20130101.12

\begin{abstract}
Peroxisomal disorders encompass a variety of pathologies with different clinical manifestations. X-linked adrenoleukodystrophy, an inherited neurodegenerative pathology, is characterized by mutation of a peroxisomal transport protein involved in the catabolism of very long-chain fatty acids (VLCFAs). These particular fatty acids abnormally accumulate in plasma and in all tissues due to the enhancement of fatty acid elongation and the impairment of fatty acid catabolism which takes place initially in the peroxisomes and afterward in the mitochondria. VLCFA accumulation cannot explain the molecular mechanisms underlying clinical manifestations in patients. Peroxisomal pathways include a product that is considered lost, but that could have a role in peroxisomal disorders: the heat produced during VLCFA catabolism. VLCFA accumulation is due to peroxisomal beta-oxidation impairment and to the enhancement of fatty acid elongation. The heat produced during peroxisomal metabolism could be a crucial factor related to the molecular mechanisms altered in X-linked adrenoleukodystrophy. VLCFA accumulation could be strongly related to the impairment of heat production, which is lost in X-linked adrenoleukodystrophy, a sort of energy necessary for the cell metabolism, which could contribute to the secondary clinical manifestations of peroxisomal disorders. Heat is not only produced during peroxisomal catabolism but also in countless metabolic pathways. It is a form of energy that could influence several parameters and could be involved in peroxisomal and metabolic disorders in general.
\end{abstract}

Keywords: Peroxisomal heat, VLCFA, Very Long-Chain Fatty Acid; X-ALD, X-linked Adrenoleukodystrophy

\section{Introduction}

Although many metabolic disorders, have been extensively studied, we receive the impression that something escapes our comprehension, something very general that is difficult to grasp. The molecular mechanisms underlying this hypothesis could be very general. For this reason, we will focus on peroxisomal molecular mechanisms and their possible relationship with peroxisomal pathologies.

Peroxisomal disorders encompass a variety of pathologies with different clinical manifestations. X-linked adrenoleukodystrophy (X-ALD) is a peroxisomal, neurodegenerative disorder, that is characterized by different phenotypes. The mutated gene ABCD1 encodes for the half-size transporter protein (ALDP), found in peroxisomes, involved in the transport and subsequent shortening of very long-chain fatty acids (VLCFAs), a process also named beta-oxidation [1-3]. In childhood cerebral X-ALD, a severe inflammatory phenotype, the abnormal accumulation of VLCFAs induces the activation of lipoxidative enzymes and inflammatory mediators, leading to neuroinflammatory demyelination [4] Metabolic alterations, associated with oxidative stress, contribute to the development of neurodegenerative processes [5].

VLCFAs are initially beta-oxidized by the peroxisomes and are further catabolized by the mitochondria, various metabolites are produced to be utilized by other metabolic pathways (e.g. ATP, a source of energy).

In X-ALD, peroxisomal VLCFA beta-oxidation is reduced, and the elongation of short fatty acids (FAs) to VLCFAs is enhanced, yielding a generally abnormal accumulation of these particularly long FAs in plasma and in all tissues of affected patients. VLCFA plasma levels are the biomarkers of X-ALD [6,7].

\section{Presentation of the Hypothesis}

VLCFA accumulation cannot really explain the molecular mechanisms underlying clinical manifestations in patients. In X-ALD, FA elongation is enhanced and VLCFA 
catabolism is reduced. Another product related to VLCFA metabolism could be, in general, the energy coming from VLCFA catabolism. This shortage could contribute to the molecular impairment of the disease.

Peroxisomes do not produce ATP, of the several metabolic pathways and products that come from the peroxisomes, a particular product is the heat, which is a form of energy. The heat produced by these organelles is considered lost [8], but this cannot be true.

\section{Implication of the Hypothesis}

In the cell, heat is generated in a variety of metabolic pathways. Although energy reserves or temperature variations are rarely considered in biological studies, they could modulate different parameters, such as tau projection domain [9]. Peroxisomal heat has been considered in relationship with catalase and thermoosmosis [10] but it could have different roles in cellular pathways.

Considering the peroxisomal disorder X-ALD and VLCFA homeostasis, the heat or energy produced during peroxisomal VLCFA catabolism could easily diffuse outside the peroxisomes, either providing input to other cellular metabolisms or being a self-sustaining product modulating VLCFA homeostasis. Peroxisomal beta-oxidation could be a heat-dependent system.

FA elongation is a complex pathway that takes place mainly in the microsomal system, and requires specific elongases. Elongase activity, enhanced in X-ALD, could require a low level of energy. In X-ALD heat could have a role in VLCFA homeostasis and have a link with the key role of acyl-CoA metabolism and with the mutated ALDP, which could be a heat-sensitive protein or a temperature stabilizer [11-15].

In general we need to improve our molecular knowledge related to heat production, and its role in cellular metabolism should be reconsidered.

In conclusion VLCFA accumulation could be strongly related to the impairment of heat production and could contribute to the secondary clinical manifestations of the peroxisomal disorder X-ALD.

\section{References}

[1] J. Mosser, A.M. Douar, C.O. Sarde, R. Feil, H. Moser, A.M. Poutska, J.L. Mandel, P. Aubourg P, "Putative X-linked adrenoleukodystrophy gene shares unexpected homology with ABC transporters," Nature 1993, 361:726-730.
[2] H.W. Moser, J. Borel, "Dietary management of X-linked adrenoleukodystrophy,” Annu Rev Nutr 1995, 15: 379-397.

[3] H.W. Moser, "Adrenoleukodystrophy: phenotype, genetics, pathogenesis, and therapy," Brain 1997, 120: 1485-1508.

[4] M. Khan, J. Singh, A.G. Gilg, T. Uto, I. Singh, "Very longchain fatty acid accumulation causes lipotoxic response via 5-lipoxygenase in cerebral adrenoleukodystrophy," J Lipid Res 2010, 51: 1685-1695.

[5] I. Singh, A. Pujol, "Pathomechanisms underlying Xadrenoleukodystrophy: a three-hit hypothesis," Brain Pathol 2010, 20:838-844.

[6] I. Singh, A.B. Moser, S. Goldfischer, H.W. Moser, "Lignoceric acid is oxidized in the peroxisomes: implication for the Zelleweger cerebro-hepatorenal syndrome and adrenoleukodystrophy,” PNAS 1984, 81: 4203-4207.

[7] S. Tsuji, T. Ohno, T. Miyatake, A. Suzuki, T. Yamakawa, "Fatty acid elongation activity in fibroblasts from patients with a drenoleukodystrophy," J Biochem 1984, 96: 12411247.

[8] H. Schulz, "Beta-oxidation in peroxisomes," In Biochemistry of Lipids, Lipoproteins and Membranes. Edited by Vance DE, Vance J. Amsterdam: Elsevier; 1996:91-93.

[9] G. Ciasca, G. Campi, A. Battisti, G. Rea, M. Rodio, M. Papi, P. Pernot, A. Tenenbaum, A. Bianconi, "Continuous thermal collapse of the intrinsically disordered protein Tau is driven by its entropi flexible domain," Langmuir 2012, 28:1340513410 .

[10] D.J. Hryb, "Peroxisomal respiration and energy conservation, possible relationship between heat production, thermoosmosis and conformational changes," FEBS Lett 1981, $128: 1-4$.

[11] H. Akanuma, Y. Kishimoto," Synthesis of ceramide and cerebroside containing both alpha-hydroxy and non hydroxy fatty acids from lignoceroyl-Coa by rat brain microsomes," J Biol Chem 1979, 254:1050-1056.

[12] A. Hlousek-Radojcic, K.J. Evenson, J.G. Jaworski, D. PostBeittenmiller, "Fatty acid elongation is indipendent of acylCoenzyme A synthetase activities in Leek and Brassica napus," Plant Phyisiol 1998, 116:251:258.

[13] P.A. Watkins, J.M. Ellis, "Peroxisomal acyl-CoA synthetases," Biochim Biophys Acta 2012, 1822:1411-1420.

[14] S. Kemp, R. Wanders, "Biochemical aspects of X-linked Adrenoleukodystrophy," Brain Pathol 2010, 20: 831-837.

[15] X. Chen, D. Shen, B. Zhou, "Analysis of the temperaturesensitive mutation of Escherichia coli pantotenate kinase reveals $\mathrm{XbjN}$ as a possible protein stabilizer," Biochem Biophys Res Commun 2006, 345: 834-842. 